\title{
Necessary and Sufficient Conditions to Linearize Doubly Recursive Programs in Logic Databases
}

\author{
WEINING ZHANG and CLEMENT T. YU \\ University of Illinois at Chicago \\ and \\ DANIEL TROY \\ Purdue University Calumet
}

\begin{abstract}
Linearization of nonlinear recursive programs is an important issue in logic databases for both practical and theoretical reasons. If a nonlinear recursive program can be transformed into an equivalent linear recursive program, then it may be computed more efficiently than when the transformation is not possible. We provide a set of necessary and sufficient conditions for a simple doubly recursive program to be equivalent to a simple linear recursive program. The necessary and sufficient conditions can be verified effectively.
\end{abstract}

Categories and Subject Descriptors: H.2.4 [Database Management]: Systems-query pracessing; I.2.3 [Artificial Intelligence]: Deduction and Theorem Proving-logic programming

General Terms: Algorithms, Theory

Additional Key Words and Phrases: Equivalence, knowledge database, linear and nonlinear recursive rules, logic database

\section{INTRODUCTION}

Processing recursive queries efficiently has been a central issue of research in the deductive database area. Numerous methods $[1,3-11,13-15,17,19,21,23-$ $25,31,33,35]$ have been proposed to evaluate recursive queries. These methods differ from each other in the types of queries that they can evaluate and in the efficiency with which they process queries. Since whether a query can be processed by a given method is determined by the syntactical structure of the query (i.e., the structure of logic rules defining the query), two equivalent queries (in terms of the answers on all possible database states) may be processed by using different evaluation methods with significantly different efficiencies. We consider two

\footnotetext{
Authors' addresses: W. Zhang and C. Yu, Department of Electrical Engineering and Computing Science, University of Chicago, Chicago, IL. 60680; D. Troy, Department of Mathematical Science, Purdue University Calumet, Hammond, IN. 46323.

Permission to copy without fee all or part of this material is granted provided that the copies are not made or distributed for direct commercial advantage, the ACM copyright notice and the title of the publication and its date appear, and notice is given that copying is by permission of the Association for Computing Machinery. To copy otherwise, or to republish, requires a fee and/or specific permission.
}

(C) 1990 ACM 0362-5915/90/0900-0459 $\$ 01.50$ 
types of queries, linear and nonlinear recursive queries, and study the characteristics of nonlinear recursive programs which can be transformed into equivalent linear recursive programs.

Motivations to study the linearization of nonlinear recursive programs include the following. From a practical point of view, strategies designed to solve linear recursions $[3-6,10,11,13-15,26,29,35]$ are usually more efficient than those proposed for solving general recursions [4, 5, 8, 10, 23, 25, 29, 31, 33]. By transforming a nonlinear recursive program into an equivalent linear one, queries can be answered with a higher efficiency by evaluating the transformed linear program. For example, a common evaluation method for answering a general recursive query is the Naive method $[4,10]$. But if a query is known to be linear recursive, we can apply the Semi-Naive $[4,10]$ method to evaluate it. It is well known, since the Semi-Naive method avoids duplicate computations, that it is likely to be more efficient than the Naive method. Notice that although the SemiNaive method can also be used for processing nonlinear recursive queries, it is usually not as efficient for an equivalent linear recursive query. Another example is given in [29], where a nonlinear recursive program is used to show that when it is processed with the Generalized Magic Set method [5], one can obtain a set of nonregular magic rules whose evaluation is as complicated as that of the original program. On the other hand, this program does have an equivalent linear recursive counterpart which can be processed efficiently by one of the methods designed to solve linear recursions.

From a theoretical point of view, it is a fundamental issue to distinguish the "easy" or "simple" linearizable recursive programs from the "difficult" or "complicated" nonlinearizable recursive programs. It is important to understand the complexity of processing recursive queries in logic databases. Similar results for the distinction between "simple" (tree) and "complicated" (cyclic) relational queries have been obtained [34].

There are several papers $[7,18,25,28]$ related to our work. The problem of deciding the equivalence of two arbitrary Horn clause logic programs has been shown to be unsolvable in [28]. In [25], a method is proposed to transform a given logic program into an equivalent one by eliminating redundant predicates and redundant rules. This is a polynomial space algorithm. Our work differs from theirs. First, we transform a simple nonlinear recursive program into a linear program by using a simple, natural conversion method to study the conditions under which the two programs are equivalent. Therefore, the rules to be considered are not as arbitrary as those in [28]. Secondly, the conditions that we obtain for linearizing doubly recursive programs can be effectively verified.

Beeri et al. [7] indicate that whether a program containing only binary chain rules is equivalent to a program containing only regular (left or right) linear binary chain rules is undecidable. The programs considered in [7] can be mutually recursive, with a recursion order greater than two, and contain more than one recursive rule. The programs that we consider are directly, doubly recursive and have only two rules (one recursive and one nonrecursive). Further, we allow arbitrary arity of predicates, and do not require the chain property. They [7] require every rule to be a binary chain rule. Their result states that whether a chain program is equivalent to some arbitrary linear chain program is 
undecidable. Nothing has been said about whether the program is equivalent to a given linear program. Because of these differences, they [7] obtained a negative result, and we have a positive one.

In [18], an approach to linearize nonlinear recursive programs is proposed. The method is based on algebra and the power-subassociativity property of bilinear functions. Their [18] result is more general than ours, since they consider mutually recursive predicates. They do not, however, provide a practical procedure. Thus the complexity of verifying their conditions for a given program is not clear. On the other hand, our result provides an effective way of identifying linearizable doubly recursive programs. Furthermore using our result, some doubly recursive programs are linearizable but do not satisfy their conditions. For example, based on our result, the program given in Example 3.6 is linearizable but the corresponding bilinear function is not power-subassociative, and therefore cannot be linearized by using the method in [18].

In this paper we consider a class of nonlinear recursive programs, called simple doubly recursive programs, and provide a set of necessary and sufficient conditions for such programs which are equivalent to the linear recursive programs obtained through a simple linearization method. A preliminary version of this paper [37] has been published in ACM SIGMOD 87. In Section 2 of this paper we provide definitions and a brief review of our previous result. In Section 3 we provide a set of necessary and sufficient conditions for a simple doubly recursive program to be linearizable and a number of examples to illustrate the result. A sketch of the proof of the conditions is given in Section 4. A proof of sufficiency is given in the Appendix.

\section{DEFINITIONS AND PREVIOUS RESULT}

A logic database consists of two components: the extensional database (EDB) containing a set of base (i.e., stored) relations (predicates); and the intensional database (IDB) containing a set of deductive rules in the form of Horn clauses. The EDB and IDB partition all predicates in the logic database, so that all derived predicates are in the IDB and all base predicates are in the EDB. Throughout this paper, we use the terms relation and predicate indistinguishably.

A rule is a function-free Horn clause of the form $B:-A_{1} A_{2} \cdots A_{k}$, where $B$ and the $A_{i}$ 's are predicates; $B$ is the head of the rule and the conjunction of $A_{i}$ 's is the body of the rule. A rule is directly recursive if the head predicate $B$ also appears in the body. Under certain restrictions, any program can be converted into an equivalent one that only contains directly recursive rules [28]. Such a transformation may not always result in an efficient program. A simple recursive program contains exactly two rules with the same head: an exit rule, which is not recursive, and a directly recursive rule. The order of recursion for a rule is the number of occurrences of the head predicate $B$ in the body of the rule. If a recursive rule has an order of recursion $R=1$, it is a linear recursive rule; if $R>1$, it is a nonlinear recursive rule. If $R=2$, it is a doubly recursive rule. In this paper we consider simple doubly recursive programs only.

In the following we define various terms that we used to formulate programs and to present our results. We will use the following example to illustrate various concepts. 
Example 2.1 (Taken from [32]). Consider a directed graph with colored arcs. Assume that there are two base relations redarc and bluearc such that $\operatorname{redarc}(x y) /$ bluearc $(x, y)$ means that the edge from a node $x$ to a node $y$ is $\mathrm{red} / \mathrm{blue}$. The following program defines paths consisting of alternating red and blue arcs, beginning and ending with a red arc.

$r_{e}: \operatorname{path}\left(x_{1} x_{2}\right):-\operatorname{redarc}\left(x_{1} x_{2}\right)$

$r_{r}: \operatorname{path}\left(x_{1} x_{2}\right):-\operatorname{path}\left(x_{1} u_{1}\right)$ bluearc $\left(u_{1} u_{2}\right)$ path $\left(u_{2} x_{2}\right)$.

The meaning of this program is that there is a path from a node $x_{1}$ to a node $x_{2}$ when there is a red arc from $x_{1}$ to $x_{2}$, or there exist some nodes $u_{1}$ and $u_{2}$ such that there are paths from $x_{1}$ to $u_{1}$ and from $u_{2}$ to $x_{2}$, as well as a blue arc from $u_{1}$ to $u_{2}$. The first rule is an exit rule and the second a recursive one.

Given a rule, all variables appearing in the head are called distinguished variables; all variables appearing in the body only are called nondistinguished variables. Let $X$ be a vector of $n$ distinguished variables and $U$ a vector of $p$ nondistinguished variables. For every predicate of arity $g$ in the body of the rule, $g$ variables are selected from the $(n+p)$ variables in $X$ and $U$ and placed at $g$ argument positions of the predicate. Such selection and placement can be represented by a formal matrix product $(X, U) H$, where $H$, called a selector, is an $(n+p) \times g(0,1)$ matrix with exactly one 1 in each column. We usually write $(X, U) H$ as $X U H$. For predicates in the body of the recursive rule, a position is distinguished or nondistinguished when it is occupied by a distinguished or nondistinguished variable. A position for a selector $H$ is the position where $H$ places a selected variable.

Example 2.1 (Continued). Consider the recursive rule for paths. The variables $x_{1}$ and $x_{2}$ are distinguished and $u_{1}$ and $u_{2}$ are nondistinguished. So $X=$ $\left(x_{1} x_{2}\right)$ and $U=\left(u_{1} u_{2}\right)$. Let us call the selectors associated with path $\left(x_{1} u_{1}\right)$, bluearc $\left(u_{1} u_{2}\right)$, and path $\left(u_{2} x_{2}\right) Z^{1}, W$, and $Z^{2}$, respectively. The matrices of the selectors are the following:

$$
Z^{1}=\left[\begin{array}{ll}
1 & 0 \\
0 & 0 \\
0 & 1 \\
0 & 0
\end{array}\right], \quad W=\left[\begin{array}{ll}
0 & 0 \\
0 & 0 \\
1 & 0 \\
0 & 1
\end{array}\right], \quad Z^{2}=\left[\begin{array}{ll}
0 & 0 \\
0 & 1 \\
0 & 0 \\
1 & 0
\end{array}\right] .
$$

It is easy to see that path $\left(X U Z^{1}\right)$ yields path $\left(x_{1} u_{1}\right)$. We say that $Z^{1}$ places $x_{1}$ to position 1 and $u_{1}$ to position 2 . The distinguished position for $Z^{1}$ is position 1 and the nondistinguished position for $Z^{1}$ is position 2. The descriptions for $W$ and $Z^{2}$ are similar.

Let $s$ be a derived predicate of arity $n$. For a given EDB, a tuple $A=$ $\left(a_{1} a_{2} \cdots a_{n}\right)$ is in $s$ with all $a$ 's constants if there is a finite derivation tree (as defined in [37] similar to the rule/goal tree in [31]), such that its root is labeled by $s(A)$, its internal nodes are labeled by tuples in $s$, and all of its leaf nodes unify with tuples in EDB. The label of any internal node is obtained from some rule defining $s$; labels of the child nodes of the internal node are described as follows. First, the predicates in the body of the rule are unified with the labels of the internal node's child nodes. The resulting substitution is then propagated to 


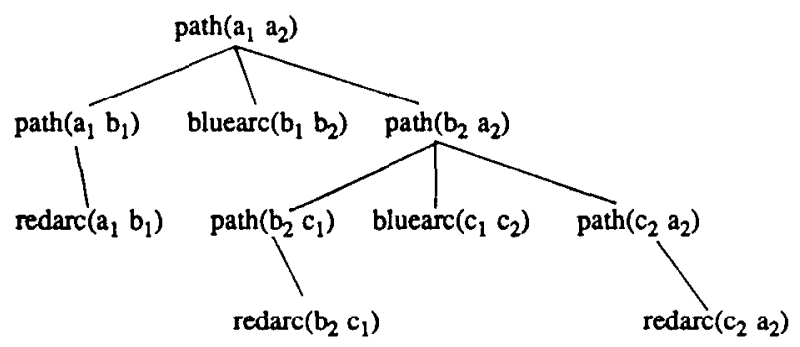

Fig. 1. Derivation tree for Example 2.1.

the head of the rule. The tuple obtained in the head is then the label of the internal node.

Example 2.1 (Continued). Assume that the EDB contains tuples redarc $\left(a_{1} b_{1}\right)$, redarc $\left(b_{2} c_{1}\right)$, redarc $\left(c_{2} a_{2}\right)$, bluearc $\left(b_{1} b_{2}\right)$, and bluearc $\left(c_{1} c_{2}\right)$, with $a$ 's, $b$ 's, and $c$ 's being nodes in the graph. Figure 1 shows a derivation tree of path $\left(a_{1} a_{2}\right)$.

Let $s(X)$ and $t(X)$ be two predicates with the same arity, defined over the same domain. We say that $s(X)$ implies $t(X)$, denoted by $s(X) \Rightarrow t(X)$, if for any given $\mathrm{EDB}$, every tuple in $s$ is also in $t$; and we say that $s(X)$ and $t(X)$ are equivalent, denoted $s(X) \Leftrightarrow t(X)$, iff $s(X) \Rightarrow t(X)$ and $t(X) \Rightarrow s(X)$.

Example 2.1 (Continued). The same type of path can also be defined by the following program.

$$
\begin{aligned}
& r_{e}: \operatorname{path}\left(x_{1} x_{2}\right):-\operatorname{redarc}\left(x_{1} x_{2}\right) \\
& r_{r}: \operatorname{path}\left(x_{1} x_{2}\right):-\operatorname{redarc}\left(x_{1} u_{1}\right) \text { bluearc }\left(u_{1} u_{2}\right) \operatorname{path}\left(u_{2} x_{2}\right) .
\end{aligned}
$$

It is not difficult to see that the path relation here is exactly the same as that defined by the previous program. That is, they are equivalent.

It is important to notice that the first program in Example 2.1 is doubly recursive, while the second one is linear recursive. The only difference between them is that in the body of the recursive rules, the first predicate of the nonlinear rule is path $\left(x_{1} u_{1}\right)$, while that of the linear rule is redarc $\left(x_{1} u_{1}\right)$. This suggests a very simple and natural way to convert a simple doubly recursive program into a simple linear recursive program. That is, to unify one of the two recursive predicates in the body of the doubly recursive rule with the head of the exit rule. We are, therefore, interested in obtaining conditions under which a given simple doubly recursive program is equivalent to its converted linear one.

To study the problem, a general notion of simple doubly recursive programs and their converted linear programs is needed. For this purpose, we generalize the path programs, and consider the following general programs, which define predicates $s$ and $s_{1}$, respectively.

$$
\begin{aligned}
& r_{e}: s(X):-f(X) \\
& r_{r}: s(X):-s\left(X U Z^{1}\right) r(X U W) s\left(X U Z^{2}\right) \\
& r_{e}: s_{1}(X):-f(X) \\
& r_{r}: s_{1}(X):-f\left(X U Z^{1}\right) r(X U W) s_{1}\left(X U Z^{2}\right)
\end{aligned}
$$


In these programs, $r_{e}$ is an exit rule; $r_{r}$ is a recursive rule; $s$ and $s_{1}$ are recursive predicates; $f$ and $r$ are distinct base predicates; $X$ and $U$ are vectors of distinguished and nondistinguished variables, respectively; and $Z^{1}, Z^{2}$, and $W$ are selectors. Notice that $s$ and $s_{1}$ should be considered as having the same predicate name. But, for convenience, they are named differently. The recursive rule in program (II) is obtained from that in program (I) by unifying the first occurrence of the $s$ predicate in the body with the head of the exit rule. As a result, the first occurrence of $s$ is replaced by an $f$ predicate. The choice of the first occurrence of $s$ is made arbitrarily. The result of choosing the second occurrence is similar. If the variables in the base predicate $f$ are in different order from those in $s$, then a new base predicate $f^{\prime}$ can be introduced such that the exit rule relates $s$ with $f^{\prime}$ and with the variables in both predicates in the same order while another rule relates $f^{\prime}$ and $f$. Thus, we can place the exit rule in the form as described in (I). Notice that although the conversion described here is simple and natural, it may not be the only possible way of obtaining linear rules from nonlinear ones. In this paper, however, we consider only the simple conversion.

Consider the recursive rule in programs (I) and (II). A variable is dangling if it is nondistinguished and selected by exactly one of the selectors $Z^{1}, Z^{2}$, and $W$. In other words, a dangling variable is not shared by two or more predicates. A position is dangling if it is occupied by a dangling variable. A variable is single (multiple) dangling if it is dangling with exactly (more than) one occurrence(s). A position is single (multiple) dangling if it is occupied by a single (multiple) dangling variable.

Example 2.2. Consider the following rule:

$$
s\left(x_{1} x_{2} x_{3} x_{4}\right):-s\left(x_{1} u_{1} x_{3} u_{2}\right) r\left(u_{1} u_{3} u_{3} u_{4}\right) s\left(u_{4} x_{2} u_{5} x_{4}\right)
$$

The variables $u_{2}$ and $u_{5}$ are single dangling and the variable $u_{3}$ is multiple dangling. The positions taken by these variables are single and multiple dangling, respectively.

In a recursive rule, let $Z^{j}$ and $Z^{k}$ be selectors of two predicates with the same predicate name, say $s$, in the body. Let the arity of $s$ be $n . Z^{j}$ is said to dominate $Z^{k}$ if, for every position $i, 1 \leq i \leq n$, the variable placed at position $i$ by $Z^{k}$ is either the same as that placed at position $i$ by $Z^{j}$, or a dangling variable that can only take those positions taken by this variable placed at $i$ by $Z^{j}$. Two selectors $Z^{j}$ and $Z^{k}$ are equivalent if they dominate each other.

Intuitively, if in the body of a rule there are two predicates with the same predicate name and the selector of one predicate dominates that of the other, then the former predicate will be stricter than the latter. The latter predicate is, therefore, redundant. Thus, if in the doubly recursive rule $r_{r}$ one of the selectors $Z^{1}$ or $Z^{2}$ dominates the other, the $s$ predicate with the dominated $Z$ selector can be discarded because, in any derivation tree, whenever the recursive rule is used to establish some tuple, the tuple satisfying the $s$ node with the dominating $Z$ always satisfies the $s$ node with the dominated $Z$ selector. If in rule $r_{r} Z^{1}$ and $Z^{2}$ are equivalent, either one can be discarded, yielding an equivalent linear recursive rule. This type of doubly recursive rule is called trivially linearizable. 


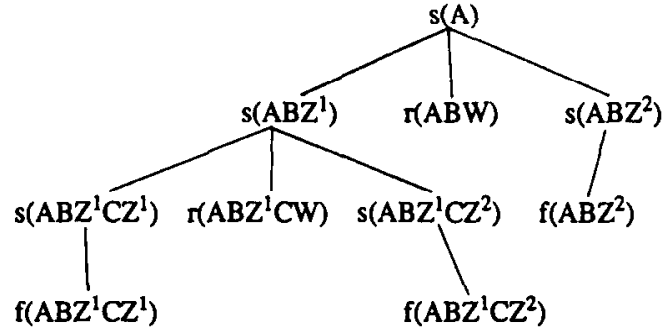

Fig. 2. Minimal derivation tree for five leaf nodes.

If in rule $r_{r}$ one of $s\left(X U Z^{1}\right)$ and $s\left(X U Z^{2}\right)$ is exactly the same as $s(X)$, the rule is called endless-recursive because, from the viewpoint of a top-down evaluation, such a rule implies an infinite recursion. The recursive rule $r_{r}$ in a simple recursive program is degenerative if it is endless-recursive or trivially linearizable.

In our previous work [30,37], we provided necessary and sufficient conditions for a simple doubly recursive program defined by (I) to be equivalent to a simple linear recursive program defined by (II), under the following assumptions:

Assumption 1. The rules are range-restricted, i.e., in each rule every distinguished variable appears in the body. This assumption is commonly used [4] to ensure finite answers to queries defined over these rules.

Assumption 2. The recursive rule $r_{r}$ is of the following type. There exist two nondistinguished variables, $u_{i}$ and $u_{j}$, such that $u_{i}$ is shared by $Z^{1}$ and $W$, but not selected by $Z^{2} ; u_{j}$ is shared by $Z^{2}$ and $W$, but not selected by $Z^{1}$. (This is a condition chaining the variables among the predicates in the body of the rule.)

Assumption 3. None of the $Z$ selectors places any distinguished variable to two or more positions in the same predicate.

Assumption 4. The tree in Figure 2 is a minimal derivation tree (in terms of the total number of nodes) of the tuple $s(A)$ for the EDB consisting of precisely the five leaf nodes, where $A, B$, and $C$ are constant vectors with distinct elements.

A tuple derived by applying the nonlinear rule once can be derived by applying the corresponding linear rule. Figure 2 gives a derivation of a tuple derived by applying the nonlinear rule twice. Assumption 4 says that there is no smaller tree to derive the tuple. In other words, the nonlinear rule is not "obviously" equivalent to a linear rule. Our intention is to identify nonlinear rules that are not obviously equivalent, but are actually equivalent to linear rules.

The previous results of [30] and [37] are summarized as follows:

Proposition 2.1. Assume that $s$ and $s_{1}$ are defined by (I) and (II), respectively, with nondegenerative recursive rules, and that they satisfy Assumptions 1 through 4. A necessary and sufficient condition for $s \Leftrightarrow s_{1}$ is that in rule $r_{r}$ the following occur:

(i) each distinguished variable $x_{j}$ is selected by at least one of the selectors, $Z^{1}$ or $Z^{2}$, and that $x_{j}$ is placed by the selector at position $j$; and

(ii) if $x_{j}$ is selected by $W$, then both $Z^{1}$ and $Z^{2}$ select $x_{j}$. 
In [30], we also give necessary and sufficient conditions for higher order recursive rules to be equivalent to linear recursive rules under rather restricted conditions.

\section{GENERALIZED NECESSARY AND SUFFICIENT CONDITIONS}

Assumptions 2 through 4 in the previous section impose some syntactic constraints on the type of doubly recursive programs to which our previous result applies. While some interesting programs do satisfy these assumptions, there are also interesting programs that do not. (For example, the well-known ancestor predicate does not satisfy Assumption 2.) In order to generalize the previous result so that it can be applied to more general types of programs, it is important to remove Assumptions 2 through 4. Thus, in this section, we only require Assumption 1 to be satisfied by programs (I) and (II), and we provide necessary and sufficient conditions for program (I) to be equivalent to linear program (II). The result is given below.

Proposition 3.1. Let $s$ and $s_{1}$ be defined by (I) and (II), respectively, with nondegenerative recursive rules that satisfy Assumption 1. Then $s \Leftrightarrow s_{1}$ iff one of the following groups of conditions is satisfied by the recursive rule.

Group 1

(1) for every distinguished variable $x_{i}$ selected by $Z^{1}$ or $Z^{2}$, an $x_{i}$ is placed at position $i$ by the $Z$ selector;

(2) every $x_{i}$ placed at more than one position by $Z^{1}$ or $Z^{2}$ is selected by both $Z^{1}$ and $Z^{2}$; and

(3) one of the following cases holds:

(a) a $W$-selector does not exist (i.e., the rule has no $r$ predicate); or

(b) $W$ exists but selects no distinguished variable; or

(c) $W$ selects some distinguished variable, shares some nondistinguished variables with $Z^{1}$ or $Z^{2}$, and for every $x_{i}$ selected by $W, x_{i}$ is selected by $Z^{1}$ and $Z^{2}$ as well; or

(d) $W$ selects some distinguished variable and shares no nondistinguished variable with $Z^{1}$ or $Z^{2}$; there is at least one distinguished variable $x_{j}$ that is selected only by $W$ and $Z^{2}$; and for every $x_{i}$ selected by $W$, one of the following cases holds:

(i) $x_{i}$ is also selected by $Z^{2}$;

(ii) each of $Z^{1}$ and $Z^{2}$ places some single dangling variable at position $i$; or

(e) $W$ selects some distinguished variable and shares no nondistinguished variable with $Z^{1}$ or $Z^{2}$; there is no distinguished variable selected by $W$ and $Z^{2}$ only; and for every $x_{i}$ selected by $W$, one of the following cases holds:

(i) both $Z^{1}$ and $Z^{2}$ place $x_{i}$ at position $i$;

(ii) both $Z^{1}$ and $Z^{2}$ place the same $x_{t}$, for some $t \neq i$, at position $i$;

(iii) each of $Z^{1}$ and $Z^{2}$ places, at position $i$, some dangling variable. 
In the last case, both variables must have the same number of occurrences and occupy the same set of positions; furthermore, for each such position $q, x_{q}$ is selected only by $W$.

\section{Group 2}

(1) $Z^{1}$ selects all distinguished variables; and

(2) (i) for every $x_{i}$ placed at some position $t$ by $Z^{1}, Z^{1}$ places $x_{t}$ at position $i$; or

(ii) $Z^{2}$ selects all distinguished variables, and for every $x_{i}$ placed at some position $t$ by $Z^{1}, Z^{2}$ places $x_{t}$ at position $i$; in either case, $Z^{1}$ places at least one $x_{i}$ to a position $t \neq i$.

\section{Group 3}

(1) for every distinguished variable $x_{i}$ selected by $Z^{1}$, an $x_{i}$ is placed at position $i$ by $Z^{1}$; and

(2) one of the following cases holds:

(i) $Z^{1}$ does not share any nondistinguished variable with $W$ or $Z^{2}$; or

(ii) $Z^{2}$ does not share any nondistinguished variable with $W$ or with $Z^{1}$, and every $x_{i}$ selected by $W$ is selected by $Z^{1}$; or

(iii) $W$ does not share any nondistinguished variable with $Z^{2}$ or with $Z^{1}$, and every $x_{i}$ selected by $Z^{2}$ is selected by $Z^{1}$.

\section{Group 4}

(1) for every distinguished variable $x_{i}$ selected by $Z^{1}$ and placed at some position $t$, there exists some position $h$ such that $Z^{1}$ also places an $x_{i}$ at $h$ and $Z^{2}$ places an $x_{h}$ at $t$; and

(2) for every nondistinguished variable $u_{s}$ shared by $Z^{1}$ and $W$, or by $Z^{1}$ and $Z^{2}$, $Z^{1}$ places a $u_{s}$ at some position $t$, then there exists some position $h$ such that $Z^{1}$ also places a $u_{s}$ at $h$ and $Z^{2}$ places an $x_{h}$ at $t$; and

(3) for every dangling variable $u_{s}$ placed at a set of positions $t$ by $Z^{1}$, cither $Z^{2}$ places a variable at all positions in $t$, or $Z^{2}$ places at each position in $t$ some distinguished variable $x_{h}$ such that $h \in t$.

\section{Group 5}

(1) for every distinguished variable $x_{i}$ selected by $Z^{1}$ and placed at some position $t$, there exists a position $h$ such that $Z^{1}$ places an $x_{i}$ at $h$ and $Z^{2}$ places an $x_{h}$ at $t$; and for every $x_{j}$ selected by $Z^{2}$ and placed at some position $p$, there exists a position $q$ such that $Z^{1}$ places an $x_{j}$ at $q$ and places an $x_{q}$ at $p$; and

(2) for every dangling variable $u_{s}$ placed at a set of positions $t$ by $Z^{1}$ (respectively ' $Z^{2}$ ), $Z^{2}$ (respectively $Z^{1}$ ) places a variable at all positions in $t$; and

(3) for every nondistinguished variable $u_{s}$ shared by $Z^{1}$ and $Z^{2}$, let a $u_{s}$ be placed at some position $t_{1}$ by $Z^{1}$ and at some position $t_{2}$ by $Z^{2}$, then either $t_{1}=t_{2}$, or there is another nondistinguished variable $u_{g}$ such that a $u_{g}$ is placed at $t_{2}$ by $Z^{1}$ and at $t_{1}$ by $Z^{2}$; and

(4) $W$ does not share any nondistinguished variable with $Z^{1}$ or with $Z^{2}$. 
Notice that the results given here are symmetric in terms of $Z^{1}$ and $Z^{2}$ if the linear recursive rule is obtained by unifying the second occurrence of $s$ in the body of the doubly recursive rule with the head of the exit rule.

Intuitively, Group 1 requires that if a distinguished variable $x$ appears in one of the recursive predicates in the body of the rule, it should be at its natural position, which is the position that it takes at the head predicate. In cases where $x$ appears in predicate $r$, both $Z^{1}$ and $Z^{2}$ will make sure that the variables placed at the natural position of $x$ are symmetric in nature. This symmetry is the key for the given program to be linearizable.

The intuitive meanings of Groups 2 through 5 can best be explained by the derivation tree in Figure 3, where only selectors are shown in the predicates.

Group 2 indicates that either $s\left(Z^{1} Z^{1}\right)$ or $s\left(Z^{1} Z^{2}\right)$ is the same as $s(X)$. Group 3 ensures that $s\left(Z^{1} Z^{1}\right)$ has the same variable pattern (in terms of which distinguished and shared nondistinguished variables are selected, where these variables are placed and how repeated variables are placed) as $s\left(Z^{1}\right)$. Group 4 ensures that $s\left(Z^{1} Z^{2}\right)$ has the same variable pattern as $s\left(Z^{1}\right)$. Group 5 implies that $s\left(Z^{1} Z^{1}\right)$ has the same variable pattern as $s\left(Z^{1}\right)$ and that $s\left(Z^{1} Z^{2}\right)$ has the same variable pattern as $s\left(Z^{2}\right)$.

Intuitively, these groups of conditions make it possible to reconstruct derivation trees in a linear fashion. This can be seen in the proof of sufficiency of the conditions.

Since the result is complicated, we demonstrate it by a number of examples.

Example 3.1. Consider the ancestor relation, defined by the following program:

$$
\begin{aligned}
& r_{e}: \text { ancestor }\left(x_{1} x_{2}\right):-\operatorname{parent}\left(x_{1} x_{2}\right) \\
& r_{r}: \text { ancestor }\left(x_{1} x_{2}\right):-\operatorname{ancestor}\left(x_{1} u_{1}\right) \text { ancestor }\left(u_{1} x_{2}\right) .
\end{aligned}
$$

The converted linear program is given below:

$$
\begin{aligned}
& r_{e}: \text { ancestor }\left(x_{1} x_{2}\right):-\operatorname{parent}\left(x_{1} x_{2}\right) \\
& r_{r}: \text { ancestor }\left(x_{1} x_{2}\right):-\operatorname{parent}\left(x_{1} u_{1}\right) \text { ancestor }\left(u_{1} x_{2}\right) .
\end{aligned}
$$

The recursive rules satisfy conditions 1, 2, and 3a of Group 1 in Proposition 3.1 (where condition 2 is trivially satisfied, since no $x_{i}$ is placed at more than one position by $Z^{1}$ or $Z^{2}$ ). Thus the two programs are equivalent. This equivalence can be easily verified by the content of the programs.

Example 3.2. Consider the path programs given in Example 2.1:

$$
\begin{aligned}
& r_{e}: \operatorname{path}\left(x_{1} x_{2}\right):-\operatorname{redarc}\left(x_{1} x_{2}\right) \\
& r_{r}: \operatorname{path}\left(x_{1} x_{2}\right):-\operatorname{path}\left(x_{1} u_{1}\right) \text { bluearc }\left(u_{1} u_{2}\right) \text { path }\left(u_{2} x_{2}\right) . \\
& r_{e}: \operatorname{path}\left(x_{1} x_{2}\right):-\operatorname{redarc}\left(x_{1} x_{2}\right) \\
& r_{r}: \operatorname{path}\left(x_{1} x_{2}\right):-\operatorname{redarc}\left(x_{1} u_{1}\right) \text { bluearc }\left(u_{1} u_{2}\right) \operatorname{path}\left(u_{2} x_{2}\right) .
\end{aligned}
$$

The recursive rules satisfy conditions 1,2 , and $3 \mathrm{~b}$ of Group 1 in Proposition 3.1. Again, condition 2 is satisfied because there is no $x_{i}$ taking more than one position in any predicate in the body of the recursive rules. Thus, by Proposition 3.1, the two programs are equivalent. Equivalence can also be verified by the content of the programs. 


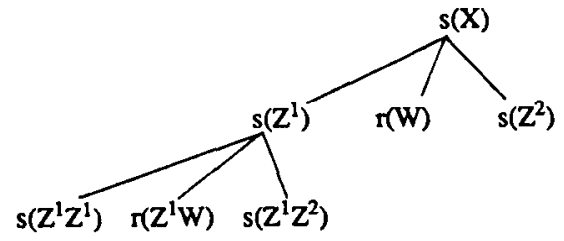

Fig. 3. Derivation tree to illustrate Groups 2-5.

Example 3.3. Let $s$ and $s_{1}$ be defined by the following programs:

$$
\begin{aligned}
& r_{e}: s\left(x_{1} x_{2} x_{3} x_{4} x_{5} x_{6} x_{7}\right):-f\left(x_{1} x_{2} x_{3} x_{4} x_{5} x_{6} x_{7}\right) \\
& r_{r}: s\left(x_{1} x_{2} x_{3} x_{4} x_{5} x_{6} x_{7}\right):-s\left(x_{1} x_{1} u_{1} u_{1} x_{5} u_{3} x_{7}\right) r\left(x_{2} x_{3} x_{4} x_{5}\right) s\left(x_{1} x_{1} u_{2} u_{2} x_{5} x_{6} u_{3}\right) . \\
& r_{e}: s_{1}\left(x_{1} x_{2} x_{3} x_{4} x_{5} x_{6} x_{7}\right):-f\left(x_{1} x_{2} x_{3} x_{4} x_{5} x_{6} x_{7}\right) \\
& r_{r}: s_{1}\left(x_{1} x_{2} x_{3} x_{4} x_{5} x_{6} x_{7}\right):-f\left(x_{1} x_{1} u_{1} u_{1} x_{5} u_{3} x_{7}\right) r\left(x_{2} x_{3} x_{4} x_{5}\right) s_{1}\left(x_{1} x_{1} u_{2} u_{2} x_{5} x_{6} u_{3}\right) .
\end{aligned}
$$

Rule $r_{r}$ satisfies conditions 1, 2, and 3e of Group 1. Rule 3e is satisfied because $W$ does not share any nondistinguished variable with the $Z$ 's, and, for the distinguished variables selected by $W, x_{5}$ satisfies condition $3 \mathrm{e}(\mathrm{i}) ; x_{2}$ satisfies $3 \mathrm{e}$ (ii); and $x_{3}$ and $x_{1}$ satisfy $3 \mathrm{e}$ (iii). By Proposition $3.1, s \Leftrightarrow s_{1}$.

Example 3.4. In this example we consider Ackerman's function. Given $0 \leq x \leq N_{x}$ and $0 \leq y \leq N_{y}$, Ackerman's function can be defined as follows:

$$
A(x, y)= \begin{cases}1, & \text { if } x=0 \\ 2, & \text { if } x=1 \text { and } y=0 \\ x+2, & \text { if } x>1 \text { and } y=0 \\ A(A(x-1, y), y-1), & \text { if } x>0 \text { and } y>0\end{cases}
$$

We define two base relations $f$ and $r$ such that $f$ contains only $f(0,0,1)$, $f(0,1,1), \ldots, f\left(0, N_{y}, 1\right), f(1,0,2), f(2,0,4), \ldots, f\left(N_{x}, 0, N_{x}+2\right)$ and $r$ contains only $r(1,1,0,0), r(1,2,0,1), \ldots, r\left(N_{x}, N_{y}, N_{x}-1, N_{y}-1\right)$. Let $s\left(x_{1} x_{2} x_{3}\right)$ state that the value of Ackerman's function of inputs $x_{1}$ and $x_{2}$ is $x_{3}$. The nonlinear program defining Ackerman's function is given below:

$$
\begin{aligned}
& r_{e}: s\left(x_{1} x_{2} x_{3}\right):-f\left(x_{1} x_{2} x_{3}\right) \\
& r_{r}: s\left(x_{1} x_{2} x_{3}\right):-s\left(u_{1} x_{2} u_{2}\right) r\left(x_{1} x_{2} u_{1} u_{3}\right) s\left(u_{2} u_{3} x_{3}\right)
\end{aligned}
$$

The converted linear program is

$$
\begin{aligned}
& r_{e}: s\left(x_{1} x_{2} x_{3}\right):-f\left(x_{1} x_{2} x_{3}\right) \\
& r_{r}: s\left(x_{1} x_{2} x_{3}\right):-f\left(u_{1} x_{2} u_{2}\right) r\left(x_{1} x_{2} u_{1} u_{3}\right) s\left(u_{2} u_{3} x_{3}\right) .
\end{aligned}
$$

Notice that the recursive rules do not satisfy condition 1 of Group 1 because of the treatment of $x_{1}$ and $x_{2}$. They do not satisfy condition 1 of Group 2 because of the placement of $x_{1}$ and $x_{3}$. They do not satisfy condition 2 of Group 3 due to the placement of $u_{1}$ and $u_{3}$. They do not satisfy condition 1 of Groups 4 and 5 because of the placement of $x_{2}$ and $u_{3}$. So for every group in Proposition 3.1, some conditions are violated, and therefore the two programs are not equivalent. In fact, the tuple $(2,2,4)$ can be derived for $s$ but not for $s_{1}$. 
Example 3.5. Let $s$ and $s_{1}$ be defined by the following programs:

$$
\begin{aligned}
& r_{e}: s\left(x_{1} x_{2} x_{3} x_{4}\right):-f\left(x_{1} x_{2} x_{3} x_{4}\right) \\
& r_{r}: s\left(x_{1} x_{2} x_{3} x_{4}\right):-s\left(x_{3} x_{1} x_{4} x_{2}\right) r\left(x_{4} x_{3}\right) s\left(x_{2} x_{4} x_{1} x_{3}\right) . \\
& r_{e}: s_{1}\left(x_{1} x_{2} x_{3} x_{4}\right):-f\left(x_{1} x_{2} x_{3} x_{4}\right) \\
& r_{r}: s_{1}\left(x_{1} x_{2} x_{3} x_{4}\right):-f\left(x_{3} x_{1} x_{4} x_{2}\right) r\left(x_{4} x_{3}\right) s_{1}\left(x_{2} x_{4} x_{1} x_{3}\right) .
\end{aligned}
$$

The recursive rule satisfies the conditions of Group 2, thus $s \Leftrightarrow s_{1}$ by Proposition 3.1 .

Example 3.6. Let $s$ and $s_{1}$ be defined by the following programs:

$$
\begin{aligned}
& r_{e}: s\left(x_{1} x_{2} x_{3}\right):-f\left(x_{1} x_{2} x_{3}\right) \\
& r_{r}: s\left(x_{1} x_{2} x_{3}\right):-s\left(u_{1} u_{2} x_{3}\right) r\left(u_{3} x_{1}\right) s\left(u_{3} u_{4} x_{2}\right) . \\
& r_{e}: s_{1}\left(x_{1} x_{2} x_{3}\right):-f\left(x_{1} x_{2} x_{3}\right) \\
& r_{r}: s_{1}\left(x_{1} x_{2} x_{3}\right):-f\left(u_{1} u_{2} x_{3}\right) r\left(u_{3} x_{1}\right) s_{1}\left(u_{3} u_{4} x_{2}\right) .
\end{aligned}
$$

Rule $r_{r}$ satisfies the conditions of Group 3 in Proposition 3.1, so $s \Leftrightarrow s_{1}$.

\section{SKETCH OF THE PROOF}

Because the proof of Proposition 3.1 is very complicated, we will only provide a sketch. A detailed proof of sufficiency for the conditions of Group 1 is given in the Appendix, and interested readers may refer to [36] and [38] for a complete proof.

Proof of Proposition 3.1 (Sufficiency). Intuitively, the proof consists of reconstructing derivation tree $T_{1}$ into one of the derivation trees $T_{2}, T_{3}$, or $T_{4}$ (see Figure 4). Under Group 1 conditions, the reconstructed tree is $T_{4}$; under Group 2 conditions, the reconstructed tree is $T_{2}$; under conditions in Groups 3, 4, or 5 , the reconstructed tree is $T_{3}$. Under Group 1 conditions, the reconstructed tree, $T_{4}$, is of the same size as the original tree, while under the conditions of the other groups, the reconstructed tree is smaller.

Condition Group 1. Assume that condition Group 1 in Proposition 3.1 is satisfied by a recursive rule. The proof consists of two steps: First, we show that for any EDB, any tuple $s(A)$, for some constant vector $A=\left(a_{1} \ldots a_{n}\right)$, derivable by the tree $T_{L}$ in Figure $5 \mathrm{a}$ is also derivable by the tree $T_{R}$ in Figure $5 \mathrm{~b}$. This is done by instantiating each $x_{i}$ (the vector of distinguished variables $\left.X=\left(x_{1}, \ldots, x_{i}, \ldots, x_{n}\right)\right)$ in $T_{R}$ to $a_{i}$ and showing that $U=\left(u_{1}, \ldots, u_{p}\right)$ and $V=\left(v_{1}, \ldots, v_{p}\right)$ nondistinguished variables can be instantiated so as to create the following unifications:

$$
\begin{aligned}
& U 1: s\left(X U Z^{1}\right) \text { unifies with } s\left(A B Z^{1} C Z^{1}\right) \\
& U 2: s\left(X U Z^{2} V Z^{1}\right) \text { unifies with } s\left(A B Z^{1} C Z^{2}\right) \\
& U 3:
\end{aligned}
$$

Depending on the conditions satisfied by the given recursive rule, these instantiations also decide for each $r$-node shown in $T_{R}$ which of $r(A B W)$ and $r\left(A B Z^{1} C W\right)$ in $T_{L}$ to unify with. Here, $B=\left(b_{1} \cdots b_{p}\right)$ and $C=\left(c_{1} \cdots c_{p}\right)$ are constant vectors defined by the given EDB; $T_{1}, T_{2}$, and $T_{3}$ are finite subtrees of any shape deriving $s\left(A B Z^{1} C Z^{1}\right), s\left(A B Z^{1} C Z^{2}\right)$, and $s\left(A B Z^{2}\right)$, respectively. 


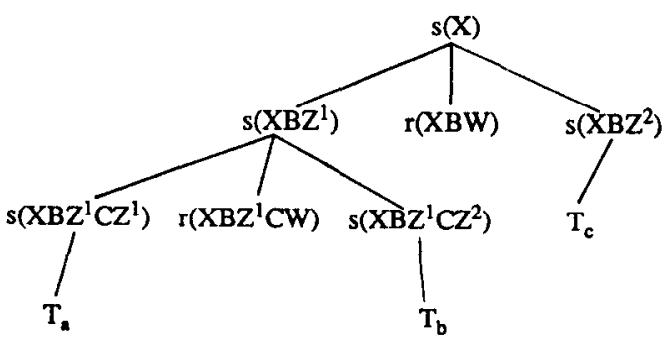

(a)

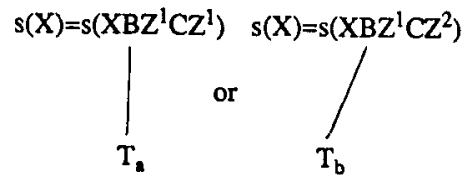

(b)

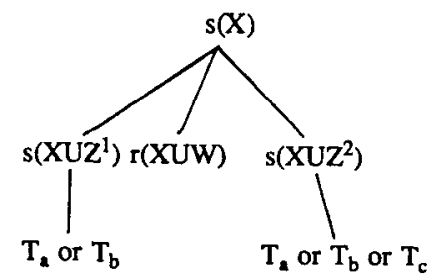

(c)

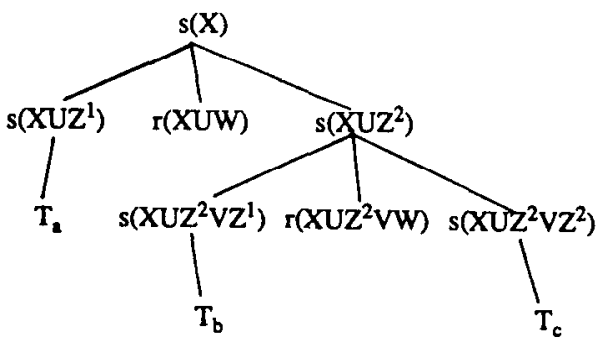

(d)

Fig. 4. Derivation trees: (a) $T_{1}$; (b) $T_{2}$; (c) $T_{3}$; and (d) $T_{4}$.

Secondly, we show that for any tuple $s(A)$ derivable for a given EDB, its derivation tree (of any shape) can be converted into an equivalent one (i.e., it still derives $s(A)$ ) by repeatedly replacing subtrees of the shape of $T_{L}$ by the corresponding $T_{R}$. The resulting tree has the following property: for every $s$ node, either the node itself is derived by an $f$ tuple (via the exit rule), or its left child (which is also an $s$ node) is derived by an $f$ tuple. It is trivial to convert such a tree into an $s_{1}$-tree deriving $s_{1}(A)$.

In the first step, we show the following:

(1) If conditions 1,2 , and 3 a are satisfied, unifications $U 1$ through $U 3$ are consistent, that is, no variable is substituted by more than one constant.

(2) If conditions 1,2 , and $3 \mathrm{~b}$ are satisfied, then not only $U 1$ through $U 3$ but also two more unifications are consistent, $U 4: r(X U W)$ unifies with $r\left(A B Z^{1} C W\right)$ and $U 5: r\left(X U Z^{2} V W\right)$ unifies with $r(A B W)$. 


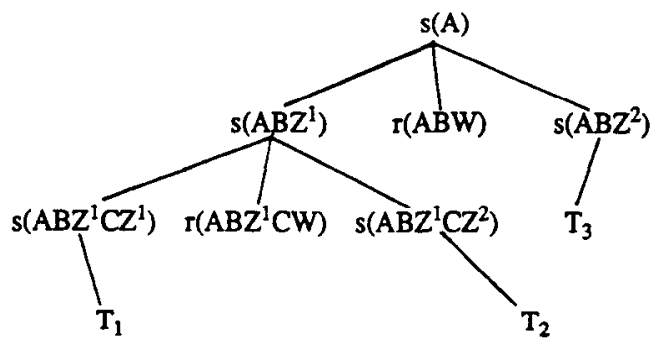

(a)

Fig. 5. Derivation trees: (a) $T_{\mathrm{L}}$; (b) $T_{\mathrm{R}}$.

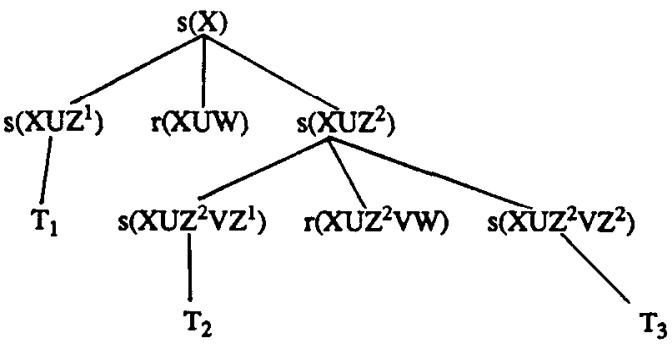

(b)

(3) If conditions 1,2 , and $3 \mathrm{c}$ are satisfied, unifications $U 1$ through $U 5$ are consistent. $U 4: r(X U W)$ unifies with $r\left(A B Z^{1} C W\right)$ and $U 5: r\left(X U Z^{2} V W\right)$ unifies with $r(A B W)$.

(4) If conditions 1,2 , and $3 \mathrm{~d}$ are satisfied, $U 1$ through $U 5$ are consistent. $U 4$ : $r(X U W)$ unifies with $r(A B W)$ and $U 5: r\left(X U Z^{2} V W\right)$ unifies with $r(A B W)$.

(5) If conditions 1,2 , and $3 \mathrm{e}$ are satisfied, $U 1$ through $U 5$ are consistent. $U 4: r(X U W)$ unifies with $r(A B W)$ and $U 5: r\left(X U Z^{2} V W\right)$ unifies with $r\left(A B Z^{1} C W\right)$.

Once we show the above, we can provide a procedure to transform a given derivation tree of arbitrary shape into an equivalent linear one. The details of the proof can be found in the Appendix.

Condition Groups 2 through 5. The sufficiency of the condition Groups 2 through 5 can be proved by using a similar approach. Given any EDB, the derivation tree of any tuple $s(A)$ is of the shape shown in Figure 6, where each $T_{i}$ is a subtree of any shape constructed by rules in program (I). Let the root of $T_{i}$ be $s^{i}$. To show that $s_{1}(A)$ can also be derived from the same EDB, we show that to establish $s(A)$, the root of each subtree $T_{i}$ in Figure 6 can be replaced by a node $s^{h}$ derivable from a tuple $f^{h}$ in the EDB using the exit rule only. Notice that when an $s$-node is replaced by another $s$-node, the subtree rooted at the former $s$-node is also replaced by the subtree rooted at the latter $s$-node. It is trivial to convert the resulting tree into an $s_{1}$-tree.

The proof consists of two steps. First, consider any subtree $T_{i}$ in Figure 6 such that its root $s^{i}$ has three children. We can show that if the recursive rule satisfies conditions 1 and 2(i) in Group 2, the parent of $s^{i}$ can be replaced by the left child 


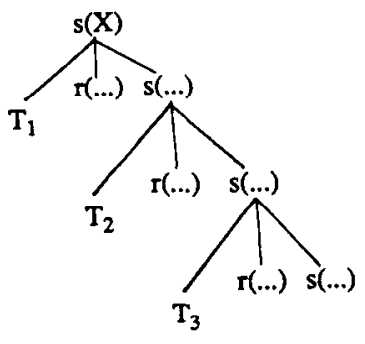

Fig. 6. General derivation tree for tuple $s(X)$.

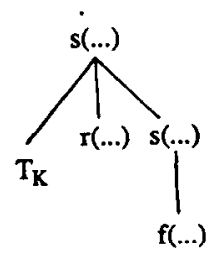

of $s^{i}$; if the rule satisfies conditions 1 and 2(ii) in Group 2, the parent of $s^{i}$ can be replaced by the right child of $s^{i}$; if the recursive rule satisfies conditions 1 and 2(i) of the Group $3, s^{i}$ can be replaced by its left child; if the rule satisfies conditions 1 and 2(ii) in Group 3, $s^{i}$ and its sibling $r$-node can be replaced by its left and middle children, respectively; if conditions 1 and 2(iii) of Group 3 are satisfied, $s^{i}$ and its sibling $s$-node can be replaced by its left and right children, respectively; if condition Group 4 is satisfied, $s^{i}$ can be replaced by its right child; and if condition Group 5 is satisfied, $s^{i}$ and its sibling $s$-node can be replaced by its right and left children, respectively. In each case, the resulting tree still derives $s(A)$.

Second, we transform the tree of $s(A)$, based on the following procedure. If the root of the tree has only one child, it can be trivially converted into a tree of $s_{1}(A)$. Otherwise, start with the left child of the root of the tree. Let us call the node currently being considered the current node. If the current node has only one child (which must be an $f$-node), then repeat the procedure on the left child of the current node's sibling $s$-node. If the current node has three children, then make the replacement based on the description given in last paragraph (the current node corresponds to $s^{i}$ ). Then repeat the procedure on the current node again. The procedure repeats until no more replacement is possible.

Since in each replacement the resulting tree is smaller than the original one while still deriving the same tuple, and the initial tree is finite for any derivable tuple in $s$, the procedure will terminate. The resulting tree is such that the left child of any $s$-node is derived by a tuple in relation $f$. This tree can be converted into an $s_{1}$-tree in an obvious way.

(Necessity). Despite the fact that we only consider simple programs with doubly recursive rules, the proof of the necessity of the five groups of conditions in Proposition 3.1 involves a complicated case analysis.

The main idea behind the proof is the following. By definition, if $s \Leftrightarrow s_{1}$, then for any EDB, a tuple derivable for $s$ implies that the same tuple must be derivable 
Fig. 7. Derivation tree $T$.

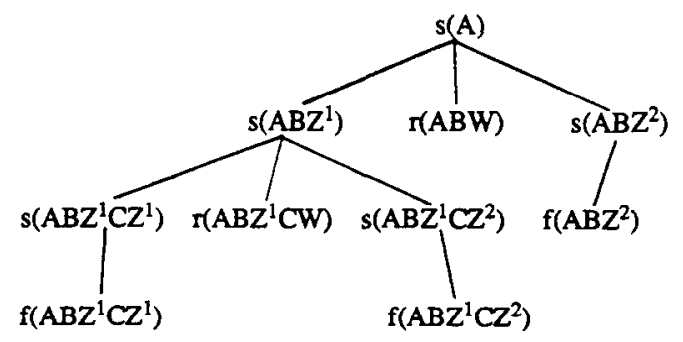

for $s_{1}$. Notice that to derive a tuple for $s_{1}$, the derivation tree must be rightskewed (or right-linear), while to derive the same tuple for $s$, the derivation tree may be left-skewed or in any other shape. So we can construct some special EDB such that a particular tuple can be derived for $s$ by a tree other than a rightskewed one. By analyzing how the same tuple can be derived for $s_{1}$ in the same $\mathrm{EDB}$, we can show that the recursive rule must satisfy one of the five groups of conditions.

The specific EDB, called DB1, is constructed from the derivation tree $T$ in Figure 7. Specifically, in DB1, there exist three constant vectors: $A=$ $\left(a_{1} a_{2} \cdots a_{n}\right), B=\left(b_{1} b_{2} \cdots b_{p}\right)$, and $C=\left(c_{1} c_{2} \cdots c_{p}\right)$ with all $a$ 's, $b$ 's, and $c$ 's being distinct. All tuples in the base relations $f$ and $r$ in DB1 are those leaf nodes of $T: f\left(A B Z^{1} C Z^{1}\right), f\left(A B Z^{1} C Z^{2}\right), f\left(A B Z^{2}\right), r\left(A B Z^{1} C W\right)$, and $r(A B W)$. Since any derivation tree of $s$ is constructed by using the rules defining $s$, the nodes (or tuples) in both $T_{L}$ and $T_{R}$ can be considered as being obtained in the same systematical way. For example, in $T_{L}$, the tuple $A B Z^{1} C Z^{1}$ is obtained as follows. First, an $n$-vector $A B Z^{1}$ is obtained by applying $Z^{1}$ to select from the concatenation of the vector $A$ of $n$ constants for distinguished variables and the vector $B$ of $p$ constants for nondistinguished variables. Then the $n$-vector $A B Z^{1}$ is concatenated with the $p$-vector $C$, and the result is selected by $Z^{1}$ again, to produce the tuple $A B Z^{1} C Z^{1}$.

It is clear that $T$ derives the tuple $s(A)$. If $s \Leftrightarrow s_{1}$, one of the derivation trees shown in Figure 8 must be the minimal tree deriving $s_{1}(A)$ by using the tuples in DB1 only, where $X=\left(x_{1} x_{2} \cdots x_{n}\right), U=\left(u_{1} u_{2} \cdots u_{p}\right)$, and $V=\left(v_{1} v_{2} \cdots v_{p}\right)$ are vectors of variables. Notice that the derivation tree $T^{*}$ in Figure 8 represents all right-linear trees that use the recursive rule at least twice.

For DB1, the establishment of a tuple in $s_{1}$ implies that there is a finite rightlinear derivation tree and a set of unifications that unify each leaf node in the $s_{1}$-tree with a tuple in the EDB such that every distinct variable is substituted by (or, equivalently, takes on) exactly one value from the constant vectors. If the tuple derived is $A$, then it is further required that each $x_{i}$ take on the value $a_{i}$. To find such a set of unifications, we consider the leaf nodes in the $s_{1}$-tree, one after another. For each leaf node, we find a tuple in the EDB such that to unify the leaf node with the tuple does not introduce any inconsistency. A unification introduces inconsistency if it substitutes a variable by using a value different from that used by some previous unification (of other leaf nodes) or it causes some leaf node to have no tuple to unify with.

In the proof, we need to consider every tree in Figure 8. If it is the minimal tree deriving $s_{1}(A)$ in $\mathrm{DB} 1$, then we need to find all possible sets of consistent 


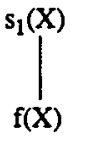

(a)

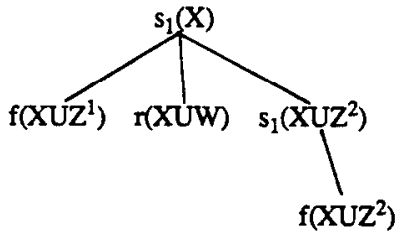

(b)

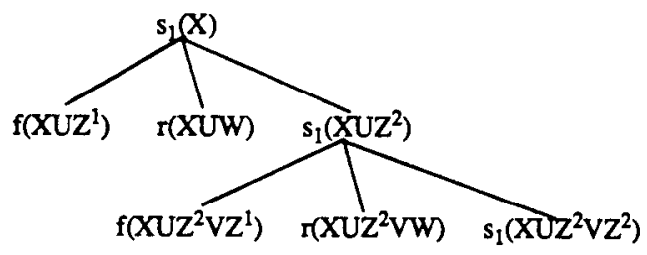

Fig. 8. Derivation tree $T^{*}$.

(c)

unifications for the leaf nodes in the tree and specify what conditions the given rule has to satisfy in order to apply each set of unifications. There are two possible situations: $T^{*}$ is or is not a minimal derivation tree of $s_{1}(A)$.

In the first situation, $T^{*}$ is the minimal derivation tree of $s_{1}(A)$. By showing that inconsistency will be introduced by various incorrect unifications, we eventually show that the node $f\left(X U Z^{1}\right)$ in $T^{*}$ can only unify with the tuple $f\left(A B Z^{1} C Z^{1}\right)$, and thus are able to claim that in DB1 we do not need to consider $T^{*}$ with height deeper than 2 . The unification determined for $f\left(X U Z^{1}\right)$ indicates that many properties are satisfied by the recursive rule. These properties can be used to determine unifications of the remaining nodes in $T^{*}$. Eventually, we can determine a unification for each node in $T^{*}$ and derive more properties. These properties result in the condition Group 1 of Proposition 3.1.

The analysis of the first situation is the most difficult part of the proof. We proved 10 lemmas and 19 sublemmas. For details, please refer to [36]. Lemma 1 indicates that if $s \Leftrightarrow s_{1}$ and $T^{*}$ is the minimal tree deriving $s_{1}(A)$ in DB1, then the recursive rule must have certain structure. Lemma 2 through Lemma 6 show various cases where inconsistency of certain unifications can be detected. Lemmas 7 and 8 show that when a given recursive rule satisfies certain properties, $s$ will not be equivalent to $s_{1}$. These two lemmas depend on Sublemma 1. Based on Lemmas 1 through 8 , Lemmas 9 and 10 show that $f\left(X U Z^{1}\right)$ does not unify with $f\left(A B Z^{2}\right)$ or $f\left(A B Z^{1} C Z^{2}\right)$. Sublemmas 2 through 19 show various properties of the recursive rule, assuming that $T^{*}$ is the minimal tree for $s_{1}(A)$ in DB1 and that certain unifications have been made (among which we have $f\left(X U Z^{1}\right)$ unified with $\left.f\left(A B Z^{1} C Z^{1}\right)\right)$.

In the second situation, derivation tree $T^{*}$ in Figure 8 is not the minimal tree for deriving $s_{1}(A)$. Thus a minimal tree of $S_{1}(A)$ must be one of the two trees (a) or (b) shown in Figure 8.

To find the necessary conditions, we need to make consistent unifications of the leaf nodes of tree (a) or (b) in Figure 8 with the leaf nodes of tree $T$. If tree 
(a) is a minimal tree of $s_{1}(A)$, we can easily show that the Group 2 conditions must be satisfied.

If tree (b) is a minimal tree of $s_{1}(A)$ in Figure 8, then because in tree (b) there are two $f$-nodes and one $r$-node, while in tree $T$ (that is in DB1) there are three tuples in relation $f$ and two tuples in relation $r$, there are altogether 18 possible ways to associate leaf nodes in tree (b) with leaf nodes in tree $T$. Since $s$ is not degenerative, it can be shown that 12 out of 18 cases are impossible. The six possible (and consistent) cases are the following:

Case 1. $f\left(X U Z^{2}\right)$ unifies with $f\left(A B Z^{2}\right), r(X U W)$ unifies with $r(A B W)$, and $f\left(X U Z^{1}\right)$ unifies with $f\left(A B Z^{1} C Z^{1}\right)$.

Case 2. $f\left(X U Z^{2}\right)$ unifies with $f\left(A B Z^{2}\right), r(X U W)$ unifies with $r(A B W)$, and $f\left(X U Z^{1}\right)$ unifies with $f\left(A B Z^{1} C Z^{2}\right)$.

Case 3. $f\left(X U Z^{2}\right)$ unifies with $f\left(A B Z^{2}\right), r(X U W)$ unifies with $r\left(A B Z^{1} C W\right)$, and $f\left(X U Z^{1}\right)$ unifies with $f\left(A B Z^{1} C Z^{1}\right)$.

Case 4. $f\left(X U Z^{2}\right)$ unifies with $f\left(A B Z^{2}\right), r(X U W)$ unifies with $r\left(A B Z^{1} C W\right)$, and $f\left(X U Z^{1}\right)$ unifies with $f\left(A B Z^{1} C Z^{2}\right)$.

Case 5. $f\left(X U Z^{2}\right)$ unifies with $f\left(A B Z^{1} C Z^{2}\right), r(X U W)$ unifies with $r(A B W)$, and $f\left(X U Z^{1}\right)$ unifies with $f\left(A B Z^{1} C Z^{1}\right)$.

Case 6. $f\left(X U Z^{2}\right)$ unifies with $f\left(A B Z^{1} C Z^{1}\right), r(X U W)$ unifies with $r(A B W)$, and $f\left(X U Z^{1}\right)$ unifies with $f\left(A B Z^{1} C Z^{2}\right)$.

For each of the six cases, a set of properties can be obtained by using techniques similar to those used in the first situation. The conditions in Group 3 are obtained by combining properties associated with Cases 1, 3, and 5; those in Group 4 are obtained from properties associated with Cases 2 and 4; and those in Group 5 are from properties associated with Case 6. The full proof is in [38].

\section{CONCLUDING REMARKS}

In this paper we extended our previous work [30, 37] by providing a set of necessary and sufficient conditions for transforming simple doubly recursive programs into their equivalent linear programs by using a simple conversion method. The results not only provide a complete characterization for a class of linearizable doubly recursive programs, but also allow for effective verification of linearizability. We believe that the linearization of nonlinear recursive programs has both practical and theoretical importance. Further research in this area will lead to fruitful results. The syntactic characterization of nonlinear rules indicates how the placement of variables in predicates affects the linearizability of a recursive rule and, in turn, how the linearizability of a recursive rule affects the complexity of recursive query processing. Such a full understanding is a foundation for efficient query processing and optimization in deductive databases. We plan to generalize our analysis to more general recursive programs. We note that the analysis is rather complicated. The complication is due to the elimination of Assumptions 2 through 4.

Saraiya indicates that he has a generalization of our result to allow multiple distinct subgoals, though no detailed proof has been provided. We have also ACM Transactions on Database Systems, Vol. 15, No. 3, September 1990. 
obtained generalizations of our results in different directions. All these are beyond the scope of this paper.

\section{APPENDIX}

In this appendix we provide a detailed proof of sufficiency for Group 1 of Proposition 3.1. As mentioned in Section 4, the proof intuitively consists of reconstructing derivation tree $T_{1}$ into one of the derivation trees $T_{2}, T_{3}$, or $T_{4}$ (see Figure 4). Under Group 1 conditions, the reconstructed tree is $T_{4}$ and the reconstructed tree is the same size as the original tree.

Assume that condition Group 1 in Proposition 3.1 is satisfied by a recursive rule. The proof consists of two steps. First, we show that for any EDB, any tuple $s(A)$, for some constant vector $A=\left(a_{1} \cdots a_{n}\right)$, derivable by the tree $T_{L}$ in Figure $5 \mathrm{a}$ is also derivable by the tree $T_{R}$ in Figure $5 \mathrm{~b}$. We do this by instantiating each $x_{i}$ (the vector of distinguished variables $X=\left(x_{1}, \ldots, x_{i}, \ldots, x_{n}\right)$ ) in $T_{R}$ to $a_{i}$ and showing that $U=\left(u_{1}, \ldots, u_{p}\right)$ and $V=\left(v_{1}, \ldots, v_{p}\right)$ nondistinguished variables can be instantiated so as to create the following unifications:

$$
\begin{aligned}
& U 1: s\left(X U Z^{1}\right) \quad \text { with } s\left(A B Z^{1} C Z^{1}\right) \text {; } \\
& U 2: s\left(X U Z^{2} V Z^{1}\right) \text { with } s\left(A B Z^{1} C Z^{2}\right) \text {; } \\
& U 3: s\left(X U Z^{2} V Z^{2}\right) \text { with } s\left(A B Z^{2}\right) \text {. }
\end{aligned}
$$

These instantiations will also unify each of the $r$-nodes shown in $T_{R}$ with one of the $r$ tuples $r(A B W)$ or $r\left(A B Z^{1} C W\right)$ in $T_{L} . B=\left(b_{1} \cdots b_{p}\right)$ and $C=\left(c_{1} \cdots c_{p}\right)$ are constant vectors defined by the given EDB; $T_{1}, T_{2}$, and $T_{3}$ are finite subtrees of any shape deriving $s\left(A B Z^{1} C Z^{1}\right), s\left(A B Z^{1} C Z^{2}\right)$, or $s\left(A B Z^{2}\right)$, respectively.

Second, we show that for any tuple $s(A)$ derivable in a given EDB, its derivation tree (of any shape) can be converted into an equivalent one (i.e., it still derives $s(A)$ ) by repeatedly replacing subtrees of the shape of $T_{L}$ by the corresponding $T_{R}$. The resulting tree has the following property: for every $s$ node, either the node itself is derived by an $f$ tuple (via the exit rule) or its left child (which is also an $s$ node) is derived by an $f$ tuple. It is trivial to convert such a tree into an $s_{1}$-tree deriving $s_{1}(A)$.

We now proceed with the first step. Consider Figure 5. As described in Section 4 , the tuples are obtained in a systematic way. Thus, to show that any tuple $s(A)$ derivable by $T_{L}$ is also derivable by $T_{R}$ with the intended unifications, we need to show that the unifications $U 1$ through $U 3$ and those for the two $r$ nodes in $T_{R}$ (to be specified later) can always be made consistently for deriving $s(A)$ (i.e., the substitution resulting from these unifications will substitute every $x_{i}$ by $a_{i}$ and each nondistinguished variable (e.g., $u_{j}$ or $v_{j}$ ) by exactly one value from the vectors $A, B$, and $C$ ). Based on condition 3 , there are five cases to consider.

Case 1. Conditions 1, 2, and $3 \mathbf{a}$ are satisfied.

Since there is no $r$-node in $T_{R}$, we only need to show that $U 1, U 2$, and $U 3$ are consistent.

First consider the distinguished variables in $T_{R}$. For every $x_{i}$ placed at some position $t$ by $Z^{1}$, an $x_{i}$ is at $t$ in $s\left(X U Z^{1}\right)$ in $T_{R}$ and, by condition 1 , an $a_{i}$ is at $t$ in $s\left(A B Z^{1} C Z^{1}\right)$ in $T_{L}$. So $U 1$ is consistent for distinguished variables. To see that $U 2$ is consistent on distinguished variables, notice that for every $x_{i}$ at some 
position $t$ in $s\left(X U Z^{2} V Z^{1}\right)$, according to the way $T_{R}$ is constructed, $Z^{2}$ must select $x_{i}$. $Z^{2}$ may or may not place $x_{i}$ at positions other than $i$. If $Z^{2}$ does not place $x_{i}$ at any other position, then having $x_{i}$ at position $t$ in $s\left(X U Z^{2} V Z^{1}\right)$ means that $Z^{1}$ selects $x_{i}$ and places it at position $t$. Notice that $t$ may or may not be the same as $i$. If $t \neq i$ (therefore $x_{t} \neq x_{i}$ ), by Assumption 1 and conditions 1 and $2, x_{t}$ is selected by $Z^{2}$ and is placed only at position $t$. Therefore $a_{i}$ is at position $t$ in $s\left(A B Z^{1} C Z^{2}\right)$. The same is true if $t=i$. If $Z^{2}$ places $x_{i}$ at some other position $p$, by Assumption 1 and condition $1, Z^{1}$ places $x_{p}$ at position $p$. By condition $2, Z^{1}$ does not place $x_{p}$ at any other position. In this case there are only two possible ways to get $x_{i}$ at position $t$ in $s\left(X U Z^{2} V Z^{1}\right)$ : either $Z^{1}$ places $x_{i}$ at $t$ or $p=t$ and $Z^{1}$ places $x_{p}$ at $t$. In both cases, $a_{i}$ is at $t$ in $s\left(A B Z^{1} C Z^{2}\right)$. Thus, $U 2$ is also consistent for distinguished variables. By a similar argument, $U 3$ is also consistent for distinguished variables.

Consider nondistinguished variables in $T_{R}$. For every nondistinguished position $p$ for $Z^{1}$ (respectively, $Z^{2}$ ), by condition 1 and Assumption $1, x_{p}$ is selected only by $Z^{2}$ (respectively, $Z^{1}$ ), and by conditions 1 and $2, x_{p}$ is placed only at position $p$. Thus, for every nondistinguished variable $u_{g}$ placed at some position $t_{1}, \ldots, t_{k}$, for some $k \geq 1$ by $Z^{1}$ (respectively $Z^{2}$ ), we have $u_{g}$ at $t_{1}, \ldots, t_{k}$ in $s\left(X U Z^{1}\right.$ ) (respectively, $\left.s\left(X U Z^{2} V Z^{1}\right)\right), v_{g}$ at $t_{1}, \ldots, t_{k}$ in $s\left(X U Z^{2} V Z^{1}\right.$ ) (respectively, $\left.s\left(X U Z^{2} V Z^{2}\right)\right)$ in $T_{R}$ and $b_{g}$ at $t_{1}, \ldots, t_{k}$ in $s\left(A B Z^{1} C Z^{2}\right)$ (respectively, $s\left(A B Z^{2}\right)$ ), $c_{g}$ at positions $t_{1}, \ldots, t_{k}$ in $s\left(A B Z^{1} C Z^{1}\right)$ (respectively, $\left.s\left(A B Z^{1} C Z^{2}\right)\right)$. Thus $U 1$ through $U 3$ substitute $u_{g}$ by $c_{g}$ and $v_{g}$ by $b_{g}$. So $U 1$ through $U 3$ are consistent for nondistinguished variables.

Case 2. Conditions 1, 2, and 3b are satisfied.

In this case the intended unification of $r$-nodes in $T_{R}$ unify $r(X U W)$ with $r\left(A B Z^{1} C W\right)$ (denoted by $U 4$ ) and $r\left(X U Z^{2} V W\right)$ with $r(A B W)$ (denoted by $U 5$ ).

Consider distinguished variables in $T_{R}$. By the same argument as in Case 1, $U 1$ and $U 3$ are consistent for distinguished variables. Since $W$ does not select distinguished variables, $U 4$ and $U 5$ are consistent for distinguished variables and, by an argument similar to that given in Case $1, U 2$ is consistent for distinguished variables.

Consider nondistinguished variables in $T_{R}$. Notice that for every nondistinguished position $p$ for $Z^{1}$ (respectively, $Z^{2}$ ), by Assumption $1, x_{p}$ is selected by at least one of $Z^{1}, Z^{2}$, and $W$. Since $p$ is nondistinguished for $Z^{1}$ (respectively, $Z^{2}$ ), by condition $1, Z^{1}$ (respectively, $Z^{2}$ ) does not select $x_{p}$, by condition $3 \mathrm{~b}, W$ does not select $x_{p}$ either. So $x_{p}$ is selected by $Z^{2}$ (respectively, $Z^{1}$ ) only, and, by conditions 1 and 2 , is placed at position $p$ only. Also notice that for every nondistinguished variable $u_{g}$ placed at some position $t$ by $W, u_{g}$ is at $t$ in $r(X U W)$ and $v_{g}$ is at $t$ in $r\left(X U Z^{2} V W\right)$ in $T_{R}^{\prime}$, while $b_{g}$ is at $t$ in $r(A B W)$ and $c_{g}$ is at $t$ in $r\left(A B Z^{1} C W\right)$. By arguments similar to those given in Case $1, U 1$ through $U 5$ are consistent on nondistinguished variables.

Case 3. Conditions 1, 2 and $3 \mathrm{c}$ are satisfied.

In this case, the intended unifications of the $r$ nodes in $T_{R}$ are to unify $r(X U W)$ with $r\left(A B Z^{1} C W\right)$ (denoted by $\left.U 4\right)$ and $r\left(X U Z^{2} V W\right)$ with $r(A B W)$ (denoted by $U 5$ ). We show that unifications $U 1$ through $U 5$ can be made consistently. 
Consider distinguished variables in $T_{R}$. By an argument similar to that given in Case 1, $U 1$ through $U 3$ are consistent on distinguished variables. To see that $U 4$ and $U 5$ are also consistent on distinguished variables, notice that by conditions 1 and $3 \mathrm{c}$, in $T_{R}$, if $x_{i}$ is at a position $t$ in $r(X U W)$ then it is also at $i$ in $s\left(X U Z^{2}\right)$, and therefore is also at $t$ in $r\left(X U Z^{2} V W\right)$. This also means that $a_{i}$ is at $t$ in both $r$ tuples in $T_{L}$.

Now consider nondistinguished variables in $T_{R}$. Since in this case, for every nondistinguished position $t$ for $Z^{2}$ (respectively, $Z^{1}$ ), by Assumption 1 and conditions 1,2 , and $3 \mathrm{c}, x_{t}$ is selected by $Z^{1}$ (respectively, $Z^{2}$ ) only and placed at position $t$ only. Thus a similar argument as given in Case 2 can be used to show that $U 1$ through $U 5$ are consistent on nondistinguised variables, too.

Case 4. Conditions 1, 2, and $3 \mathrm{~d}$ are satisfied.

In this case, the intended unifications for $r$ nodes in $T_{R}$ are to unify both $r(X U W)$ and $r\left(X U Z^{2} V W\right)$ with $r(A B W)$ (denoted by $U 4$ and $U 5$, respectively).

Consider distinguished variables in $T_{R}$. By using the same arguments as used in Case 1, we can show that conditions 1 and 2 assure that $U 1, U 2$, and $U 3$ are consistent for distinguished variables. It is trivial to see that $U 4$ is also consistent for distinguished variables. Let us consider $r\left(X U Z^{2} W\right)$. For every $x_{i}$ placed at some position $t$ by $W, a_{i}$ is at $t$ in $r(A B W)$ in $T_{L}$. So $U 5$ implies that $a_{i}$ will substitute for the variable at position $i$ in $s\left(X U Z^{2}\right)$ in $T_{R}$. By condition $3 \mathrm{~d}$, the variable at position $i$ in $s\left(X U Z^{2}\right)$ is either $x_{i}$ or a single dangling variable. In either case, $U 5$ is consistent for distinguished variables.

Consider nondistinguished variables in $T_{R}$. Because $W$ does not share a nondistinguished variable with $Z^{1}$ or $Z^{2}$, for every nondistinguished variable $u_{g}$ selected by $W, U 4$ and $U 5$ are consistent on $u_{g}$ and $v_{g}$ in $T_{R}$. For every nondistinguished position $p$ for $Z^{1}$ (respectively, $Z^{2}$ ), by Assumption 1, conditions 1 , 2 , and $3 \mathrm{~d}, x_{p}$ is ( 1 ) selected by $Z^{2}$ (respectively, $Z^{3}$ ) only and placed at position $p$ only; or (2) selected by $W$ only and $p$ is a single dangling position for both $Z^{1}$ and $Z^{2}$. Notice that (1) and (2) cannot both be true. For those nondistinguished variables which are placed at positions where (1) is true, arguments similar to those given in Case 2 can be used to show that $U 1$ through $U 5$ are consistent. For any nondistinguished position $p$ where (2) is true, let some $u_{g}$ be at $p$ in $s\left(X U Z^{2}\right)$ and some $u_{s}$ be at $p$ in $s\left(X U Z^{1}\right)$ where both $u$-variables are single dangling. Since $x_{p}$ is selected by $W$ only, let $W$ place $x_{p}$ at some position $q$. Only $r\left(X U Z^{2} V W\right)$ needs consideration for this type of nondistinguished variable. Then, $u_{g}$ is at position $q$ in $r\left(X U Z^{2} V W\right)$ while $a_{p}$ is at $q$ in $r(A B W)$ and $b_{s}$ is at $q$ in $r\left(A B Z^{1} C W\right)$. $U 5$ assures consistency on $u_{g}$. Thus $U 1$ through $U 5$ are consistent.

Case 5. Conditions 1, 2, and $3 e$ are satisfied.

The intended unifications for the $r$ nodes in $T_{R}$ are to unify $r(X U W)$ with $r(A B W)$ (denoted by $U 4$ ) and $r\left(X U Z^{2} V W\right)$ with $r\left(A B Z^{1} C W\right)$ (denoted by $U 5$ ).

Consider distinguished variables in $T_{R}$. By the same arguments as in Case 4, U1 through $U 4$ are consistent for distinguished variables. Consider $r\left(X U Z^{2} V W\right)$ in $T_{R}$. For every $x_{i}$ at some position $t$ in $r\left(X U Z^{2} V W\right)$, there must be some position $p$ such that $Z^{2}$ places $x_{i}$ at $p$ and $W$ places $x_{p}$ at $t$. As 
implied by condition $3 \mathrm{e}, Z^{1}$ also places $x_{i}$ at position $p$ (notice that $i$ and $p$ may or may not be equal). So in $T_{L}, a_{i}$ is at $p$ in $s\left(A B Z^{1}\right)$ and therefore at $t$ in $r\left(A B Z^{1} C W\right)$. This implies that $U 5$ is also consistent for distinguished variables.

Consider nondistinguished variables in $T_{R}$. Again, for every $u_{g}$ selected by $W$, $U 4$ and $U 5$ are consistent on $u_{g}$ and $v_{g}$ in $T_{R}$. For every nondistinguished position $t_{1}$ for $Z^{1}$ (respectively, $Z^{2}$ ), by Assumption 1, conditions 1, 2 and $3 \mathrm{e}$, $x_{t_{1}}$ is (1) selected by $Z^{2}$ (respectively, $Z^{1}$ ) only and placed at position $t_{1}$ only; or (2) selected by $W$ only and for both $Z^{1}$ and $Z^{2}, p$ is dangling; furthermore, if the dangling variable $u_{g}$ is placed by $Z^{1}$ (respectively, $Z^{2}$ ) at some positions $t_{1}, \ldots$, $t_{k}$, the other dangling variable $u_{s}$ must be placed by $Z^{2}$ (respectively, $Z^{1}$ ) at $t_{1}, \ldots, t_{k}$ and $x_{t_{1}}, \ldots, x_{t_{k}}$ are selected by $W$ only. Notice that (1) and (2) cannot both be true. For nondistinguished variables which are placed at positions where (1) is true, $U 1$ through $U 5$ can be shown to be consistent by arguments similar to those given for Case 2. For nondistinguished variables which are placed at positions where (2) is true, similar to Case 4 , only $r\left(X U Z^{2} V W\right)$ needs to be considered, and $U 5$ assures consistency. Thus, $U 1$ and $U 5$ are consistent.

Now let us consider the second step. For any derivation tree of any tuple $s(A)$ in a given $E D B$, we can transform it based on the following procedure:

Start at the root of the given derivation tree of $s(A)$. If the root has only one child, which must be an $f$-node, the tree can be trivially converted into a tree of $s_{1}(A)$. Otherwise, for every $s$-node, say $s^{\prime}$, which has three children, if the left child of $s^{\prime}$ is established by an $f$ tuple (via the exit rule), then repeat the procedure on the subtree rooted at the right child of $s^{\prime}$. If the left child of $s^{\prime}$ also has three children, then the subtree rooted at $s^{\prime}$ is of the shape of $T_{L}$ and can be replaced by the corresponding tree $T_{R}$. After replacing the subtree of $s^{\prime}$, the procedure is repeated on the new subtree rooted at $s^{\prime}$. The procedure repeats until no more replacements are possible.

Since each time the replacement occurs the root of the subtree (i.e., $s^{\prime}$ ) remains unchanged and the subtree with the shape of $T_{R}$ consists of the same subtrees $T_{1}, T_{2}$, and $T_{3}$ of corresponding $T_{L}$, the leaf nodes (i.e., tuples in $f$ and $r$ ) in the two trees are the same. Since for any derivable tuple the initial tree is finite, the above procedure always terminates. It is obvious that when the procedure terminates, the resulting tree derives $s(A)$, and is such that for every $s$-node in the tree, it is established for an $f$ tuple or its left child is derived by an $f$ tuple. $\square$

\section{ACKNOWLEDGMENTS}

We are grateful to Jeffrey Ullman for his comments on an earlier draft of this paper, which led to the discovery of conditions stated in Groups 2 through 5 in Proposition 3.1. We also thank the referees for their comments and suggestions. We are grateful to Won Kim for his suggestions, which led to improvements in the readability of this paper. This research was supported in part by NSF under grant IRI-8901789.

\section{REFERENCES}

1. Agrawal, R., and Devanbu, P. Moving selections into linear least fixpoint queries. In Proceedings of the IEEE Conference Data Engineers (Los Angeles, Calif., 1988), 452-461. 
2. AGRAWAL, R., AND JAGADISH, H. V. Direct algorithms for computing the transitive closure of database relations. In Proceedings of Thirteenth International Conference on Very Large Data' Bases (Brighton, England, 1987). 255-266.

3. Bancilhon, F. Naive evaluation of recursively defined relations. In On Knowledge Base Management Systems-Integrating Database and AI Systems, Brodie and Mylopoulos, Eds., Springer-Verlag, New York, 1985.

4. BANCILHON, F., AND RAMAKRISHNAN, R. An amateur's introduction to recursive query processing strategies. In Proceedings of ACM SIGMOD (Washington, D.C., 1986). ACM, New York, 1986, 16-52.

5. BeERI, C., AND Ramakrishnan, R. On the power of Magic. In Proceedings of ACM Symposium on Principles of Database Systems (San Diego, Calif., 1987). ACM, New York, 1987, 269-283.

6. Bancilhon, F., Maier, D., Sagiv, Y., AND Ullman, J. Magic sets and other strange ways to implement logic programs. In ACM Symposium on Principles of Database Systems (1986). ACM, New York, 1986.

7. Beeri, C., Kanellakis, P., Bancilhon, F., and Ramakrishnan, R. Bounds on the propagation of selection into logic programs. In Proceedings of Principles of Data Base Conference (Cambridge, Mass., 1987), 214-226.

8. Ceri, S., Gottlob, G., and Lavazza, L. Translation and optimization of logic queries: The algebraic approach. In Proceedings of Twelfth International Conference on Very Large Data Bases (Kyoto, Aug. 1986). 395-402.

9. Chakravarthy, U., Grant, J., and Minker, J. Foundations of semantic query optimization for deductive databases. In Proceedings of The Workshop on Foundations of Deductive Databases and Logic Programming (Washington, D.C., 1986).

10. Chang, C. On the evaluation of queries containing derived relations in relational databases. In Advances in Data Base Theory, Vol. 1, H. Gallaire, J. Minker, and J. Nicholas, Eds., Plenum Press, New York, 235-260, 1979.

11. GaRdarin, G. Evaluation of database recursive logic programs as recurrent function series. In Proceedings of ACM SIGMOD (Washington, D.C., 1986). ACM, New York, 1986, 117-186.

12. GARDARIN, G. Magic functions: A technique to optimize extended datalog recursive programs. In Proceedings of Thirteenth International Conference on Very Large Data Bases (Brighton, England, 1987), 21-30.

13. HAN, J., AND Henschen, L. Handling redundancy in the processing of recursive database queries. In Proceedings of ACM SIGMOD (1987). ACM, New York, 1987, 73-81.

14. HAN, J., AND LU, H. Some performance result on recursive query processing in relational database systems. In Proceedings of IEEE Data Eng. (Los Angeles, Calif., 1986), 533-539.

15. Henschen, L., AND Naqvi, S. On compiling queries in recursive first-order databases. J. ACM 31, 1 (Jan. 1984), 47-85.

16. IoANNIDIS, $Y$. A time bound on the materialization of some recursively defined views. In Proceedings of Conference on Very Large Data Bases (Stockholm, 1985), 219-226.

17. IOANNIDIS, Y., AND WoNG, E. An algebraic approach to recursive inference. In Proceedings of Conference on Expert Database Systems (Charleston, S.C., 1986), 209-223.

18. IoANNIDIS, Y., AND WoNG, E. Transforming nonlinear recursion into linear recursion. In Proceedings 2nd International Conference on Expert Database Systems (Vienna, Va., 1988), 187-207.

19. Jagadish, H. V., Agrawal, R., and Ness, L. A study of transitive closure as a recursive mechanism. In Proceedings of ACM SIGMOD (1987). ACM, New York, 1987.

20. MAIER, D. The Theory of Relational Databases. Computer Science Press, Rockville, Md., 1983.

21. Naughton, J. Data independent recursion in deductive databases. In Proceedings of Conference on Principles of Database Syst. (Cambridge, Mass., 1986), 267-279.

22. Naughton, J. One-sided recursion. In Proceedings of Conference on Principle of Database Syst. (San Diego, Calif., 1987), 340-346.

23. NEJDL, W. Recursive strategies for answering recursive queries-The RQA/FQI strategy. In Proceedings of Thirteenth International Conference on Very Large Data Bases (Brighton, England, 1987), 43-50.

24. RASCHID, L., AND SU, S. Y. W. A parallel processing strategy for evaluating recursive queries. In Proceedings of Conference on Very Large Data Bases (Kyoto, 1986), 412-419. 
25. SAGIV, Y. Optimizing datalog programs. Extended abstract. In Proceedings 6th ACM Symposium on Principles of Database Systems (1987). ACM, New York, 1987, 349-362.

26. SACCA, D., AND Zaniolo, C. On the implementation of a simple class of logic queries for databases. In ACM Symposium on Principles of Database Systems (Cambridge, Mass., 1986). ACM, New York, 1986, 16-23.

27. SaCCA, D., AND ZANiolo, C. Magic counting methods. In Proceedings of ACM SIGMOD (San Francisco, 1987). ACM, New York, 1987, 49-59.

28. SHMUELI, O. Decidability and expressiveness aspects of logic queries. In ACM Symposium on Principles of Database Systems (1987). ACM, New York, 1987, 237-249.

29. SiPPU, S., AND SoISALON-SOININEN, E. An optimization strategy for recursive queries in logic databases. Extended abstract. IEEE Data Eng. (1988), 470-477.

30. Troy, D., Yu, C., AND ZhaNG, W. Linearization of nonlinear recursive rules. IEEE Trans. Softw. Eng. 15 (Sept. 1989), 1109-1119.

31. UllmaN, J. Implementation of logical query languages for databases. ACM Trans. Database Syst. 10, 3 (Sept. 1985), 289-321.

32. Ullman, J. Principles of Database and Knowledge-Base Systems, Vol. 2, Computer Science Press, Rockville, Md., 1989.

33. VIEILlE, L. Recursive axioms in deductive databases: The query/subquery approach. Expert Database Syst. (1986), 179-193.

34. YU, C., AND OzSoYoglu, M. An algorithm for tree-query membership of a distributed query. IEEE COMPSAC (Chicago, Ill., 1979). IEEE, New York, 1979.

35. YU, C., AND ZHANG, W. Efficient recursive query processing using wavefront methods. IEEE Data Eng. (Los Angeles, Calif., 1987), 652-657.

36. Zhang, W., Yu, C., and Troy, D. Necessary and sufficient conditions to linearize doubly recursive programs in logic datahases. Tech. Rep., Dept. of EECS, Univ. of Illinois, Chicago, 1988.

37. Zhang, W., AND YU, C. A necessary condition for a doubly recursive rule to be equivalent to a linear recursive rule. In Proceedings of ACM SIGMOD (1987). ACM, New York, 1987, 345-356.

38. ZHANG, W. A linearization of nonlinear recursive programs in logic databases. Ph.D. thesis, Dept. of EECS, Univ. of Illinois, Chicago, 1988.

Received October 1988; revised January 1989 and May 1989; accepted August 1989 